\section{Fatores de risco e proteção para doenças crônicas: vigilância por meio de inquérito telefônico, VIGITEL, Brasil, 2007}

\author{
Risk and protective factors for chronic non- \\ communicable diseases: the VIGITEL telephone \\ disease surveillance system, Brazil, 2007
}

Erly Catarina Moura ${ }^{1}$

Sara Araújo da Silva 1

Deborah Carvalho Malta 1,2

Otaliba Libânio Morais Neto 1

\footnotetext{
1 Ministério da Saúde, Brasília, Brasil.

2 Escola de Enfermagem, Universidade Federal de Minas Gerais, Belo Horizonte, Brasil.

Correspondência E. C. Moura Ministério da Saúde. SQSW 504, bloco F, apto. 306, Brasília, DF 70673-506, Brasil. erlycm@usp.br
}

\begin{abstract}
This article describes the prevalence of risk and protective factors for chronic non-communicable diseases in Brazil, using data collected in 2007 through the telephone disease surveillance system (VIGITEL). We evaluated 54,252 adults residing in Brazil's State capitals and Federal District. Men showed higher rates of smoking, overweight, consumption of soft drinks and fatty meat, sufficient leisure-time physical activity, sedentary lifestyle, and binge drinking; women showed higher consumption of fruits and vegetables. For men with more schooling, the probability of smoking decreased by $42 \%$ and consumption of fatty meat decreased by 31\%; overweight increased 86\%, sedentary lifestyle $42 \%$, regular consumption of fruits and vegetables $89 \%$, and leisure-time physical activity 78\%. For women with more schooling, the probability of smoking decreased $31 \%$, overweight $26 \%$, and consumption of fatty meat 35\%; sedentary lifestyle increased 76\%, leisure-time physical activity $77 \%$, regular consumption of fruits and vegetables $48 \%$, and recommended consumption offruits and vegetables $75 \%$.
\end{abstract}

Chronic Disease; Adult; Surveillance

\section{Introdução}

As doenças crônicas não transmissíveis constituem sério problema de saúde pública, tanto nos países ricos, quanto nos de média e baixa renda. Estimativas da Organização Mundial da Saúde (OMS) apontam que essas doenças já são responsáveis por $58,5 \%$ de todas as mortes ocorridas no mundo e por $45,9 \%$ da carga global de doença 1 . No ano de 2005, cerca de 35 milhões de pessoas morreram de doenças crônicas no mundo, o que corresponde ao dobro das mortes relacionadas às doenças infecciosas 2 .

O Brasil tem experimentado, nas últimas décadas, importantes transformações no seu padrão de mortalidade e morbidade, em decorrência dos processos de transição epidemiológica, demográfica e nutricional. Em relação ao primeiro, ocorreu importante redução das doenças infecciosas e aumento das doenças crônicas não transmissíveis, bem como de acidentes e violências. Séries históricas de estatísticas de mortalidade disponíveis para as capitais dos estados brasileiros indicam que a proporção de mortes por doenças crônicas não transmissíveis aumentou em mais de três vezes entre as décadas de 1930 e de 19903.

As doenças crônicas não transmissíveis se caracterizam por terem etiologia múltipla, muitos fatores de risco, longos períodos de latência, curso prolongado, origem não infecciosa e, também, por associarem-se a deficiências e incapa- 
cidades funcionais. Dentre os fatores de risco comuns, destacamos o consumo de tabaco, de bebidas alcoólicas em excesso e de gorduras saturadas, além do excesso de peso, da inatividade física e do baixo consumo de frutas, legumes e verduras 1 . A emergência dessas enfermidades é muito influenciada pelas condições de vida, não sendo resultado unicamente de escolhas individuais; por outro lado, ao longo da vida, existem muitas oportunidades de prevenção devido à longa duração de tais doenças 2 .

A experiência de outros países mostra que o sucesso das intervenções de saúde pública, no que se refere à redução dos fatores de risco e da prevalência das doenças crônicas não transmissíveis, é maior à medida que ações de promoção da saúde e de prevenção dessas doenças e de seus fatores de risco sejam realizadas de maneira integrada e abrangente 2,4 . Essas evidências levaram a Secretaria de Vigilância a implantar o seu sistema de monitoramento de fatores de risco, que inclui, desde 2006, a Vigilância de Fatores de Risco e Proteção para Doenças Crônicas por Inquérito Telefônico (VIGITEL) 5. O VIGITEL possibilita o monitoramento desses fatores, fornecendo informações importantes, para que se planejem políticas públicas de promoção e prevenção, além de se avaliarem as intervenções realizadas 3 .

O objetivo deste artigo é descrever a prevalência de indicadores do VIGITEL avaliados em 2007, em inquérito realizado com a população adulta das 26 capitais de estados brasileiros e do Distrito Federal.

\section{Métodos}

O VIGITEL avalia anualmente pouco mais de 54 mil indivíduos com 18 anos de idade ou mais, em um mínimo de duas mil entrevistas por cidade, de modo a estimar, com coeficiente de $95 \%$ de confiança e erro máximo de cerca de dois pontos percentuais, a frequência de qualquer fator de risco. A amostragem se dá em duas etapas: seleção de linhas telefônicas residenciais fixas, valendo-se do cadastro eletrônico cedido pelas companhias telefônicas majoritárias de cada cidade, e sorteio de um morador adulto para responder a entrevista. Maiores detalhes podem ser vistos em Moura et al. 6 .

As entrevistas são realizadas por meio de questionário eletrônico, contendo questões sobre características sociodemográficas, consumo de tabaco e de bebidas alcoólicas, alimentação, peso e altura, atividade física e determinadas doenças.

As estimativas dos indicadores avaliados são ajustadas para representar a população adulta total de cada cidade e do conjunto das cidades avaliadas. Para isso, são considerados os pesos do número de adultos e de linhas telefônicas de cada residência (adultos/linhas), a razão de indivíduos, avaliados segundo a população residente em 36 categorias construídas pela combinação de sexo, faixa etária e nível de escolaridade. Considera-se, ainda, a fração amostral, dada pela razão entre o número de indivíduos do VIGITEL de cada cidade e o número de adultos residentes nela 6 .

O presente trabalho analisa as frequências de fatores de risco e proteção dos seguintes indicadores selecionados do VIGITEL: fumantes, exfumantes, excesso de peso, obesidade, consumo regular de frutas, legumes e verduras, consumo adequado de frutas, legumes e verduras, consumo regular de refrigerantes com açúcar, hábito de consumo de carne com gordura visível, atividade física suficiente no tempo livre, inatividade física, consumo abusivo de bebidas alcoólicas, referência a diagnóstico médico prévio de hipertensão arterial e diabetes.

Considerou-se ex-fumante o indivíduo que referiu já ter fumado alguma vez na sua vida e fumante aquele que fuma atualmente, independente do número de cigarros em ambos os casos, em resposta positiva respectivamente às questões “O(a) Sr(a) fuma?” " “O(a) Sr(a). já fumou?". $\mathrm{O}$ excesso de peso e a obesidade foram calculados com base em informações autorreferidas sobre peso e altura. Considerou-se excesso de peso quando o índice de massa corporal (IMC), dado pelo peso em quilos dividido pelo quadrado da altura em metros, foi $\geq 25 \mathrm{~kg} / \mathrm{m}^{2}$, e obesidade quando o IMC atingiu $\geq 30 \mathrm{~kg} / \mathrm{m}^{2}$. Consumo regular de frutas, legumes e verduras e consumo regular de refrigerante com açúcar foram considerados para a frequência $\geq 5$ dias na semana; o consumo recomendado de frutas, legumes e verduras para $\geq 5$ vezes por dia em $\geq 5$ dias na semana. $O$ hábito de consumo foi considerado presente para o relato positivo de consumo de carnes com gordura visível. Consideraram-se ativos no tempo livre aqueles que praticavam atividade vigorosa (corrida, corrida em esteira, ginástica aeróbica, futebol, basquetebol ou tênis) por $\geq 20$ minutos em $\geq 3$ dias por semana, ou atividade leve ou moderada (todas as demais modalidades, a saber: caminhada, caminhada em esteira, musculação, hidroginástica, ginástica em geral, natação, artes marciais e luta, bicicleta, voleibol e outros) por $\geq 30$ minutos em $\geq 5$ dias por semana. Inatividade física foi considerada para aqueles que não praticavam nenhuma atividade física no tempo livre, para os que autorreferiram esforço físico leve ou muito leve no trabalho, utilizavam transporte motorizado para locomoção ao trabalho e 
realizavam pouco esforço físico nas atividades domésticas. Consumo abusivo de bebidas alcoólicas foi considerado presente se foram ingeridas mais do que cinco doses em uma única ocasião nos últimos trinta dias, para homens, e mais do que quatro, para mulheres. Hipertensão arterial e diabetes foram consideradas presentes quando havia referência a diagnóstico médico prévio dessas doenças.

As frequências dos indicadores avaliados foram calculadas para o conjunto da população brasileira das 27 cidades estudadas, segundo sexo e escolaridade. Razões de prevalência ajustadas para idade da ocorrência desses indicadores foram calculadas, separadamente para homens e mulheres, segundo a escolaridade, utilizando-se regressão linear de Poisson. A análise dos dados foi realizada com o auxílio do aplicativo Stata, versão 9 (Stata Corp., College Station, Estados Unidos), considerando-se intervalos de 95\% de confiança (IC95\%) e nível de 5\% de significância.

O projeto foi aprovado pela Comissão Nacional de Ética em Pesquisa para Seres Humanos do Ministério da Saúde.

\section{Resultados}

Em 2007, o VIGITEL ligou para 138.600 números telefônicos, identificando 75.876 linhas potencialmente residenciais. Em 54.251 destas, as entrevistas foram concluídas, resultando numa taxa de sucesso de $71,5 \%$. A taxa de recusa foi de 6,6\%: 1,8\% em Palmas (Tocantins); $2,1 \%$ em Cuiabá (Mato Grosso); 2,4\% em Rio Branco (Acre); 2,5\% em Porto Velho (Rondônia); 3\% em Campo Grande (Mato Grosso do Sul); 3,1\% no Distrito Federal; 3,4\% em Goiânia (Goiás); 3,6\% em Curitiba (Paraná); 3,9\% em Boa Vista (Roraima); 4\% em Macapá (Amapá); 4,1\% em Manaus (Amazonas); 4,6\% em São Luís (Maranhão); 4,8\% em Florianópolis (Santa Catarina); 5\% em Natal (Rio Grande do Norte); 5,3\% em Belém (Pará); 5,5\% em Teresina (Piauí); 6,1\% em João Pessoa (Paraíba); 6,1\% em Maceió (Alagoas); 6,7\% em Salvador (Bahia); 7\% em Aracaju (Sergipe); 7\% em Porto Alegre (Rio Grande do Sul); 7,1\% em Belo Horizonte (Minas Gerais); 7,3\% em Fortaleza (Ceará); 7,7\% em Recife (Pernambuco); 8,7\% em Vitória (Espírito Santo); 10,4\% em São Paulo e $11 \%$ no Rio de Janeiro. A taxa de perdas foi de 21,9\%: 18,2\% em Belo Horizonte; 18,8\% em São Paulo; 19,3\% no Rio de Janeiro; 19,4\% em Recife; 19,7\% em Fortaleza; 19,9\% em Salvador; 19,9\% em Vitória; 21\% em Aracaju; 21,1\% em São Luís; 21,7\% em João Pessoa; 22,2\% em Manaus; 22,5\% em Macapá; 22,5\% em Maceió; 22,6\% em Curitiba; 23,3\% em Belém; 23,6\% em Goiânia; 23,6\% em Teresina; $24,8 \%$ no Distrito Federal; $24,9 \%$ em Natal; 24,9\% em Porto Velho; 25,2\% em Boa Vista; 25,3\% em Palmas; 26,2\% em Campo Grande; 26,2\% em Rio Branco; 27\% em Cuiabá; 29\% em Porto Alegre e $30,4 \%$ em Florianópolis.

A Tabela 1 mostra a frequência de fatores de risco e proteção para doenças crônicas não transmissíveis no conjunto da população estudada. Para todos os indicadores, notam-se, à exceção da obesidade e diagnóstico médico de diabetes, diferenças significativas entre os sexos. São mais frequentes nos homens: hábito de fumar (fumante e ex-fumante), excesso de peso, consumo regular de refrigerantes com açúcar, hábito de consumo de carnes com gordura visível, prática de atividade física suficiente no tempo livre, inatividade física no geral e consumo abusivo de bebidas alcoólicas. As mulheres têm maior frequência de consumo regular e recomendado de frutas, legumes e verduras e referem, com mais frequência, o diagnóstico médico prévio de hipertensão arterial.

Quanto à escolaridade, verifica-se que, para os homens (Tabela 2), esta se associa diretamente com excesso de peso, consumo regular e recomendado de frutas, legumes e verduras, atividade física suficiente no tempo livre e inatividade física; associa-se inversamente com hábito de fumar (fumante e ex-fumante), consumo regular de refrigerante com açúcar e hábito de consumo de carnes com gordura visível. Por sua vez, para as mulheres (Tabela 3), a escolaridade associa-se diretamente com consumo regular e recomendado de frutas, legumes e verduras, atividade física suficiente no tempo livre e inatividade física; associa-se inversamente com hábito de fumar (fumante e ex-fumante), excesso de peso, obesidade, hábito de consumo de carnes com gordura visível, consumo abusivo de bebidas alcoólicas, referência ao diagnóstico médico de hipertensão arterial e de diabetes.

A Figura 1 mostra as razões de prevalência, ajustadas para idade, de alguns fatores de risco (tabagismo, excesso de peso, consumo de carnes com gordura, abuso de bebidas alcoólicas e inatividade física), segundo anos de escolaridade, em homens e mulheres. Para os homens, a probabilidade de tabagismo, hábito de consumo de carnes com gordura e consumo abusivo de bebidas alcoólicas praticamente não se altera após o ajuste para a idade. Comparativamente ao nível de mais baixa escolaridade (0-8 anos de estudo), no nível mais alto ( $\geq 12$ anos de estudo), a probabilidade de tabagismo reduz $42 \%$ e a de consumo de carnes com gordura, $31 \%$. Todavia, as probabilidades de excesso de peso e de inatividade física aumentam, chegando a, respectivamente, $86 \%$ e $42 \%$ na faixa de maior escolaridade. 
Frequência (\%) * de indicadores de doenças crônicas não transmissíveis em adultos ( $\geq 18$ anos) nas capitais de estado e Distrito Federal, segundo sexo. Vigilância de Fatores de Risco e Proteção para Doenças Crônicas por Inquérito Telefônico (VIGITEL), Brasil, 2007.

\begin{tabular}{|c|c|c|c|c|c|c|c|}
\hline \multirow[t]{2}{*}{ Indicadores } & \multicolumn{2}{|c|}{ Total } & \multicolumn{2}{|c|}{ Homens } & \multicolumn{2}{|c|}{ Mulheres } & \multirow[t]{2}{*}{ Valor de $\mathrm{F}$} \\
\hline & $\%$ & IC95\% & $\%$ & IC95\% & $\%$ & IC95\% & \\
\hline \multicolumn{8}{|l|}{ Hábito de fumar } \\
\hline Fumante & 16,4 & $15,5-17,3$ & 20,9 & $19,4-22,3$ & 12,6 & $11,6-13,6$ & $<0,001$ \\
\hline Ex-fumante & 21,9 & $21,1-22,7$ & 25,8 & $24,4-27,2$ & 18,6 & $17,7-19,6$ & $<0,001$ \\
\hline \multicolumn{8}{|l|}{ Excesso de peso } \\
\hline Excesso de peso (IMC $\left.\geq 25 \mathrm{~kg} / \mathrm{m}^{2}\right)$ & 43,4 & $42,3-44,5$ & 49,2 & $47,5-50,9$ & 37,8 & $36,5-39,1$ & $<0,001$ \\
\hline Obesidade (IMC $\left.\geq 30 \mathrm{~kg} / \mathrm{m}^{2}\right)$ & 12,9 & $12,2-13,5$ & 13,7 & $12,7-14,8$ & 12,0 & $11,2-12,9$ & 0,159 \\
\hline \multicolumn{8}{|l|}{ Alimentação } \\
\hline Consumo de frutas, legumes e verduras $\geq 5$ dias na semana & 29,4 & $28,5-30,3$ & 22,4 & $21,1-23,6$ & 35,5 & $34,3-36,7$ & $<0,001$ \\
\hline Consumo de frutas, legumes e verduras $\geq 5$ por dia $\geq 5$ dias na semana & 13,9 & $13,2-14,6$ & 10,0 & $9,0-10,9$ & 17,3 & $16,3-18,2$ & $<0,001$ \\
\hline Consumo de refrigerante com açúcar $\geq 5$ dias na semana & 26,7 & $25,6-27,7$ & 31,7 & $30,0-33,3$ & 22,4 & $21,2-23,6$ & $<0,001$ \\
\hline Hábito de consumo de carne com gordura visível & 32,8 & $31,7-33,8$ & 42,7 & $41,0-44,3$ & 24,3 & $23,0-25,5$ & $<0,001$ \\
\hline \multicolumn{8}{|l|}{ Atividade física } \\
\hline Atividade física no tempo livre considerada suficiente ** & 15,5 & $14,8-16,3$ & 19,3 & $17,9-20,6$ & 12,3 & $11,6-13,0$ & $<0,001$ \\
\hline 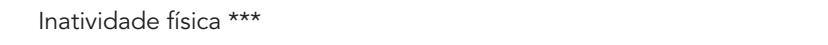 & 29,2 & $28,3-30,2$ & 30,9 & $29,4-32,5$ & 27,8 & $26,6-29,0$ & $<0,001$ \\
\hline \multicolumn{8}{|l|}{ Consumo de bebidas alcoólicas } \\
\hline Abusivo \# & 17,5 & $16,7-18,4$ & 27,2 & $25,7-28,7$ & 9,3 & $8,4-10,2$ & $<0,001$ \\
\hline \multicolumn{8}{|l|}{ Referência a diagnóstico médico } \\
\hline Hipertensão arterial & 22,9 & $22,1-23,7$ & 20,3 & $19,1-21,6$ & 25,1 & $24,0-26,2$ & $<0,001$ \\
\hline Diabetes & 5,3 & $4,9-5,7$ & 4,8 & $4,3-5,4$ & 5,7 & $5,2-6,2$ & 0,298 \\
\hline
\end{tabular}

IC95\%: intervalo de 95\% de confiança; IMC: índice de massa corporal.

* Percentual ponderado para ajustar a distribuição sociodemográfica da amostra VIGITEL à distribuição da população adulta de cada cidade no Censo Demográfico de 2000 (Instituto Brasileiro de Geografia e Estatística. http://www.ibge.gov.br) e para levar em conta o peso populacional em cada cidade;

** Atividade vigorosa (corrida, corrida em esteira, ginástica aeróbica, futebol, basquetebol ou tênis) em $\geq 20$ minutos em $\geq 3$ dias por semana, ou atividade leve ou moderada em $\geq 30$ minutos em $\geq 5$ dias por semana;

*** Nenhuma atividade física no tempo livre, autorreferência a esforço físico leve ou muito leve no trabalho, transporte motorizado e pouco esforço físico nas atividades domésticas;

\# Consumo de $>5$ doses (homem) e $>4$ doses (mulher) em $\geq 1$ dia nos últimos 30 dias.

Para as mulheres, a probabilidade de ocorrência de todos indicadores sofre alteração com o ajuste da idade: a probabilidade do tabagismo reduz em $31 \%$; a de excesso de peso, $26 \%$; de consumo de carnes com gordura, $35 \%$; já a probabilidade de inatividade física aumenta em $76 \%$.

A Figura 2 ilustra as razões de prevalência de fatores de proteção (consumo regular e recomendado de frutas, legumes e verduras e atividade física suficiente no tempo livre), também ajustadas para idade, em homens e mulheres, segundo escolaridade. Para homens, o ajuste para idade não altera a probabilidade de consumo recomendado de frutas, legumes e verduras, que chega a $89 \%$ no nível de maior escolaridade; porém, a probabilidade de consumo regular perde a associação com escolaridade, e a de atividade física aumenta para $78 \%$. Entre as mulheres, a probabilidade de atividade física no tempo livre não se altera com o ajuste da idade, sendo $77 \%$ maior no nível de mais alta escolaridade; o consumo regular de frutas, legumes e verduras chega a $48 \%$ e o recomendado, a $75 \%$.

\section{Discussão}

Uma das principais limitações do VIGITEL refere-se ao alcance da telefonia fixa, uma vez que a cobertura não é homogênea nas diferentes cidades estudadas, variando de 33,7\%, em Macapá, a 82,2\%, em Curitiba, segundo dados da Pesquisa de Orçamentos Familiares (POF) realizada entre 2002 e 2003 7. Nesse caso, a atribuição de pesos, como descrito na metodologia, tem por objetivo corrigir as sub e super-representações de de- 
Tabela 2

Frequência (\%) * de indicadores de doenças crônicas não transmissíveis em homens ( $\geq 18$ anos) nas capitais de estado e Distrito Federal, segundo escolaridade. Vigilância de Fatores de Risco e Proteção para Doenças Crônicas por Inquérito Telefônico (VIGITEL), Brasil, 2007.

\begin{tabular}{|c|c|c|c|c|c|c|c|}
\hline \multirow[t]{3}{*}{ Indicadores } & \multicolumn{6}{|c|}{ Escolaridade (anos de estudo) } & \multirow[t]{3}{*}{ Valor de $\mathrm{p}$} \\
\hline & \multicolumn{2}{|r|}{$0-8$} & \multicolumn{2}{|c|}{$9-11$} & \multicolumn{2}{|r|}{$\geq 12$} & \\
\hline & $\%$ & $\mathrm{IC} 95 \%$ & $\%$ & $\mathrm{IC} 95 \%$ & $\%$ & $\mathrm{IC} 95 \%$ & \\
\hline \multicolumn{8}{|l|}{ Hábito de fumar } \\
\hline Fumante & 25,0 & $22,5-27,5$ & 17,0 & $15,3-18,6$ & 14,8 & $12,8-16,9$ & $<0,001$ \\
\hline Ex-fumante & 29,5 & $27,1-31,9$ & 20,5 & $18,9-22,2$ & 23,3 & $21,0-25,5$ & $<0,001$ \\
\hline \multicolumn{8}{|l|}{ Excesso de peso } \\
\hline Excesso de peso (IMC $\left.\geq 25 \mathrm{~kg} / \mathrm{m}^{2}\right)$ & 48,3 & $45,5-51,2$ & 45,2 & $43,1-47,4$ & 57,9 & $55,2-60,5$ & $<0,001$ \\
\hline Obesidade (IMC $\left.\geq 30 \mathrm{~kg} / \mathrm{m}^{2}\right)$ & 13,8 & $12,1-15,5$ & 12,6 & $11,1-14,0$ & 15,3 & $13,4-17,3$ & 0,662 \\
\hline \multicolumn{8}{|l|}{ Alimentação } \\
\hline Consumo de frutas, legumes e verduras $\geq 5$ dias na semana & 18,9 & $16,9-20,9$ & 20,5 & $18,9-22,2$ & 35,8 & $33,3-38,3$ & $<0,001$ \\
\hline Consumo de frutas, legumes e verduras $\geq 5$ por dia $\geq 5$ dias na semana & 8,8 & $7,2-10,3$ & 8,4 & $7,2-9,6$ & 16,3 & $14,3-8,3$ & 0,004 \\
\hline Hábito de consumo de refrigerante com açúcar $\geq 5$ dias na semana & 32,5 & $29,7-35,3$ & 35,8 & $33,7-38,0$ & 22,2 & $20,0-24,4$ & $<0,001$ \\
\hline Hábito de consumo de carne com gordura visível & 45,5 & $42,8-48,3$ & 44,1 & $41,9-46,2$ & 32,0 & $29,6-34,4$ & $<0,001$ \\
\hline \multicolumn{8}{|l|}{ Atividade física } \\
\hline Atividade física no tempo livre considerada suficiente ** & 15,3 & $13,1-17,5$ & 22,5 & $20,7-24,4$ & 25,8 & $23,5-28,1$ & $<0,001$ \\
\hline Inatividade física $* \star \star$ & 29,2 & $26,6-31,8$ & 28,6 & $26,7-30,5$ & 40,0 & $37,3-42,6$ & 0,009 \\
\hline \multicolumn{8}{|l|}{ Consumo de bebidas alcoólicas } \\
\hline Abusivo \# & 26,5 & $24,0-29,1$ & 28,4 & $26,5-30,3$ & 27,1 & $24,8-29,4$ & 0,783 \\
\hline \multicolumn{8}{|l|}{ Referência a diagnóstico médico } \\
\hline Hipertensão arterial & 23,4 & $21,4-25,5$ & 15,0 & $13,5-16,5$ & 19,7 & $17,6-21,9$ & $<0,001$ \\
\hline Diabetes & 5,5 & $4,6-6,3$ & 3,6 & $2,8-4,3$ & 4,9 & $3,6-6,1$ & 0,091 \\
\hline
\end{tabular}

* Percentual ponderado para ajustar a distribuição sociodemográfica da amostra VIGITEL à distribuição da população adulta de cada cidade no Censo Demográfico de 2000 (Instituto Brasileiro de Geografia e Estatística. http://www.ibge.gov.br) e para levar em conta o peso populacional em cada cidade; ** Atividade vigorosa (corrida, corrida em esteira, ginástica aeróbica, futebol, basquetebol ou tênis) em $\geq 20$ minutos em $\geq 3$ dias por semana, ou atividade leve ou moderada em $\geq 30$ minutos em $\geq 5$ dias por semana;

*** Nenhuma atividade física no tempo livre, autorreferência a esforço físico leve ou muito leve no trabalho, transporte motorizado e pouco esforço físico nas atividades domésticas;

\# Consumo $>5$ doses (homem) e $>4$ doses (mulher) em $\geq 1$ dia nos últimos 30 dias.

terminadas categorias. Por exemplo, o VIGITEL, comparativamente à distribuição sociodemográfica de 2000, avalia mais mulheres, menos jovens, pessoas com mais alta escolaridade 8; após o uso dos pesos, a distribuição demográfica do VIGITEL torna-se igual à do Censo. Todavia, essa correção é parcial, pois coberturas muito baixas são consideradas comprometedoras no caso de desfechos relacionados ao nível socioeconômico, em que a posse de telefone fixo representa uma proxy. Portanto, estudos domiciliares e via celular são fundamentais para avaliar esse viés.

Ainda assim, é possível estimar as prevalências dos fatores de risco e proteção para doenças crônicas não transmissíveis para a população adulta do conjunto das cidades estudadas. Diferenças entre os sexos são encontradas para a maioria dos fatores avaliados.
O tabagismo atingiu mais os homens (20,9\%) do que as mulheres (12,6\%), mantendo a tendência já observada em 1989 - 43,3\% em homens e $27 \%$ em mulheres ${ }^{9}$-, mas com queda aproximada de $50 \%$ em ambos os sexos. Segundo a OMS 1, o uso do tabaco é o maior fator de risco prevenível de doenças crônicas não transmissíveis, conforme mostra a queda entre 1989 e 2007, que diminui com o aumento da escolaridade, tanto em homens quanto em mulheres, situação já identificada em 2003 com os dados da Pesquisa Mundial de Saúde 9.

Os homens também apresentam prevalência de excesso de peso $(49,2 \%)$ maior do que as mulheres (37,8\%), a exemplo do Inquérito Domiciliar sobre Comportamentos de Risco e Morbidade Referida de Doenças e Agravos Não Transmissíveis: Brasil, 15 Capitais e Distrito Federal, 
Frequência (\%) * de indicadores de doenças crônicas não transmissíveis em mulheres ( $\geq 18$ anos) nas capitais de estado e Distrito Federal, segundo escolaridade. Vigilância de Fatores de Risco e Proteção para Doenças Crônicas por Inquérito Telefônico (VIGITEL), Brasil, 2007.

\begin{tabular}{|c|c|c|c|c|c|c|c|}
\hline \multirow[t]{3}{*}{ Indicadores } & \multicolumn{6}{|c|}{ Escolaridade (anos de estudo) } & \multirow{3}{*}{$\begin{array}{l}\text { Valor } \\
\text { de } p\end{array}$} \\
\hline & \multicolumn{2}{|r|}{$0-8$} & \multicolumn{2}{|r|}{$9-11$} & \multicolumn{2}{|c|}{$\geq 12$} & \\
\hline & $\%$ & IC95\% & $\%$ & IC95\% & $\%$ & IC95\% & \\
\hline \multicolumn{8}{|l|}{ Hábito de fumar } \\
\hline Fumante & 14,5 & $12,8-16,2$ & 10,2 & $9,1-11,3$ & 11,0 & $9,5-12,5$ & $<0,001$ \\
\hline Ex-fumante & 21,2 & $19,6-22,8$ & 14,8 & $13,5-16,0$ & 17,4 & $15,6-19,2$ & $<0,001$ \\
\hline \multicolumn{8}{|l|}{ Excesso de peso } \\
\hline Excesso de peso (IMC $\left.\geq 25 \mathrm{~kg} / \mathrm{m}^{2}\right)$ & 45,7 & $43,4-48,0$ & 30,5 & $28,8-32,2$ & 31,2 & $29,0-33,4$ & $<0,001$ \\
\hline Obesidade (IMC $\left.\geq 30 \mathrm{~kg} / \mathrm{m}^{2}\right)$ & 16,0 & $14,4-17,6$ & 9,0 & $7,9-10,1$ & 7,5 & $6,4-8,6$ & $<0,001$ \\
\hline \multicolumn{8}{|l|}{ Alimentação } \\
\hline Consumo de frutas, legumes e verduras $\geq 5$ dias na semana & 34,2 & $32,2-36,1$ & 32,0 & $30,3-33,6$ & 45,9 & $43,6-48,2$ & 0,003 \\
\hline Consumo de frutas, legumes e verduras $\geq 5$ por dia $\geq 5$ dias na semana & 16,1 & $14,6-17,6$ & 15,3 & $14,0-16,5$ & 24,6 & $22,6-26,7$ & $<0,001$ \\
\hline Hábito de consumo de refrigerante com açúcar $\geq 5$ dias na semana & 21,7 & $19,8-23,6$ & 26,1 & $24,4-27,8$ & 17,8 & $16,0-19,7$ & 0,149 \\
\hline Hábito de consumo de carne com gordura visível & 25,8 & $23,7-27,8$ & 24,7 & $23,1-26,3$ & 18,8 & $17,0-20,6$ & $<0,001$ \\
\hline \multicolumn{8}{|l|}{ Atividade física } \\
\hline Atividade física no tempo livre considerada suficiente ** & 9,9 & $8,9-11,0$ & 13,6 & $12,5-14,8$ & 17,3 & $15,6-19,1$ & $<0,001$ \\
\hline Inatividade física $* \star \star$ & 25,9 & $23,9-27,8$ & 26,2 & $24,6-27,8$ & 36,7 & $34,5-38,9$ & 0,008 \\
\hline \multicolumn{8}{|l|}{ Consumo de bebidas alcoólicas } \\
\hline Abusivo \# & 8,2 & $6,7-9,8$ & 10,1 & $9,0-11,2$ & 11,1 & $9,6-12,6$ & 0,008 \\
\hline \multicolumn{8}{|l|}{ Referência a diagnóstico médico } \\
\hline Hipertensão arterial & 33,4 & $31,5-35,3$ & 16,2 & $14,9-17,5$ & 15,1 & $13,5-16,8$ & $<0,001$ \\
\hline Diabetes & 8,1 & $7,2-9,0$ & 3,4 & $2,7-4,1$ & 2,5 & $1,8-3,2$ & $<0,001$ \\
\hline
\end{tabular}

* Percentual ponderado para ajustar a distribuição sociodemográfica da amostra VIGITEL à distribuição da população adulta de cada cidade no Censo Demográfico de 2000 (Instituto Brasileiro de Geografia e Estatística. http://www.ibge.gov.br) e para levar em conta o peso populacional em cada cidade;

** Atividade vigorosa (corrida, corrida em esteira, ginástica aeróbica, futebol, basquetebol ou tênis) em $\geq 20$ minutos em $\geq 3$ dias por semana, ou atividade leve ou moderada em $\geq 30$ minutos em $\geq 5$ dias por semana;

*** Nenhuma atividade física no tempo livre, autorreferência a esforço físico leve ou muito leve no trabalho, transporte motorizado e pouco esforço físico nas atividades domésticas;

\# Consumo $>5$ doses (homem) e $>4$ doses (mulher) em $\geq 1$ dia nos últimos 30 dias.

2002-2003 10, que apontou mais excesso de peso em homens $(39,5 \%)$ do que em mulheres $(30,6 \%)$. Estas diferenças entre sexo podem ser mais bem compreendidas no âmbito das questões culturais que fazem, por exemplo, com que as mulheres busquem um padrão de beleza e magreza, sejam mais cuidadosas na dieta, especialmente as de classe socioeconômica mais alta (neste caso, tendo a escolaridade como proxy da renda). Para os homens, a escolaridade se associa diretamente com excesso de peso; para as mulheres, inversamente, aumentando em $86 \%$ na faixa de maior escolaridade entre os homens e reduzindo em $26 \%$ entre as mulheres. O mesmo achado foi encontrado em estudo desenvolvido no Canadá 11 .

Para as mulheres, a escolaridade se associa inversamente com obesidade. Inquéritos na- cionais, como o Estudo Nacional sobre Despesa Familiar (ENDEF) 1974/1975, Pesquisa Nacional sobre Saúde e Nutrição (PNSN) 1989 e Pesquisa sobre Padrões de Vida (PPV) 1996/1997, indicaram a mesma situação observada atualmente 12 .

As mulheres são mais cuidadosas com a alimentação do que os homens: apresentam maior consumo de frutas, legumes e verduras, menor consumo de refrigerantes com açúcar e de carnes com gordura visível. Dados da Pesquisa Mundial de Saúde de 200313 também demonstraram maior consumo de frutas, legumes e verduras entre as mulheres, assim como relatado em outros países 14,15. O consumo de carnes com gordura visível atingiu 42,7\% dos homens e $24,3 \%$ das mulheres, achado consistente com os dados da POF, a qual aponta aumento de cerca de $4 \%$, entre 1974 e 2003, na participação relativa das 
Figura 1

Razão de prevalência (RP) *, ajustada para a idade, de fatores de risco selecionados segundo escolaridade em homens e mulheres ( $\geq 18$ anos) nas capitais de estado e Distrito Federal. Vigilância de Fatores de Risco e Proteção para Doenças Crônicas por Inquérito Telefônico (VIGITEL), Brasil, 2007.

a) Homens

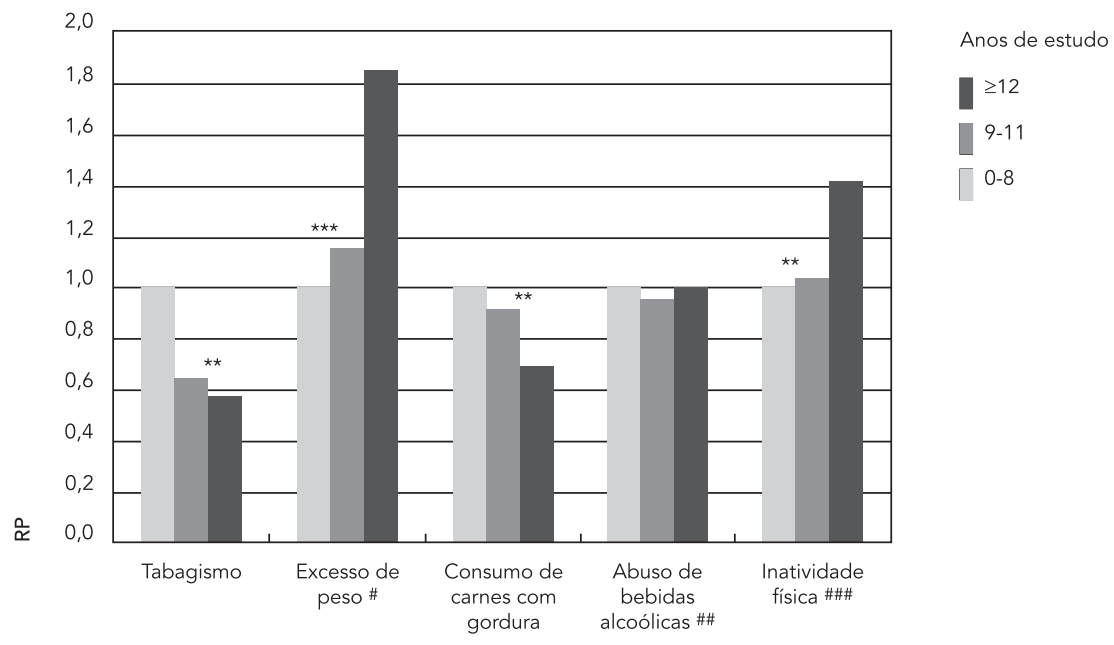

b) Mulheres

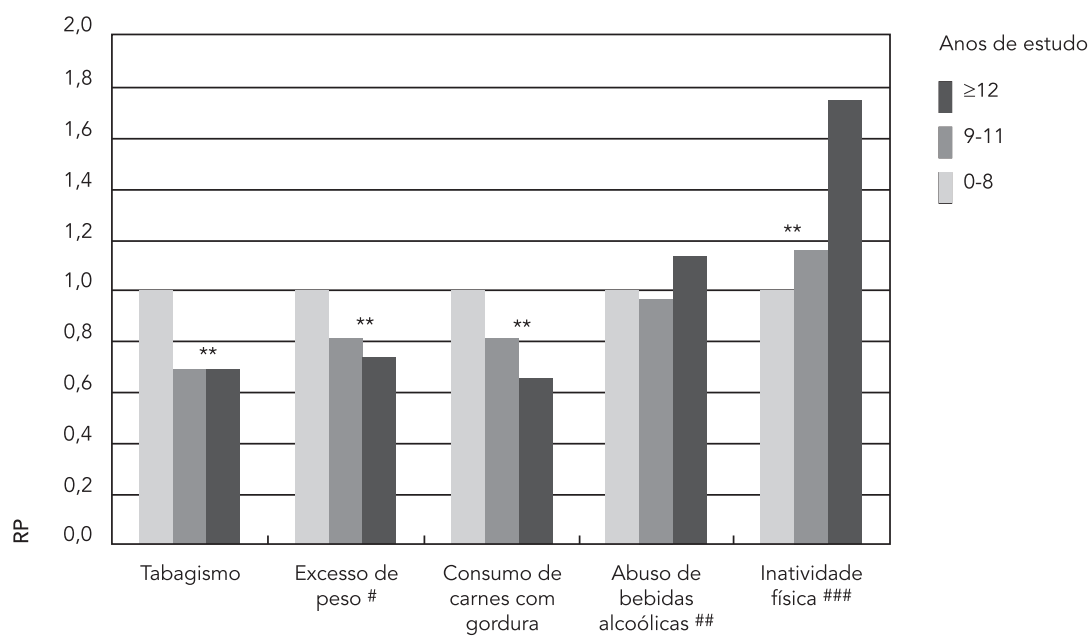

* Ponderado para ajustar a distribuição sociodemográfica da amostra VIGITEL à distribuição da população adulta de cada cidade no Censo Demográfico de 2000 (Instituto Brasileiro de Geografia e Estatística. http://www.ibge.gov.br) e para levar em conta o peso populacional em cada cidade;

$\star \star *<0,05$;

$\star \star * * 0,001$

\# Excesso de peso: índice de massa corporal (IMC) $\geq 25 \mathrm{~kg} / \mathrm{m}^{2}$;

\#\# Abuso de bebidas alcoólicas: $>5$ doses (homem) e > 4 dose (mulher) em $\geq 1$ dia nos últimos 30 dias;

\#\#\# Inatividade física: nenhuma atividade física no tempo livre, autorreferência a esforço físico leve ou muito leve no trabalho, transporte motorizado e pouco esforço físico nas atividades domésticas. 


\section{Figura 2}

Razão de prevalência (RP) *, ajustada para a idade, de fatores de proteção selecionados, segundo escolaridade em homens e mulheres ( $\geq 18$ anos) nas capitais de estado e Distrito Federal, segundo sexo. Vigilância de Fatores de Risco e Proteção para Doenças Crônicas por Inquérito Telefônico (VIGITEL), Brasil, 2007.

a) Homens

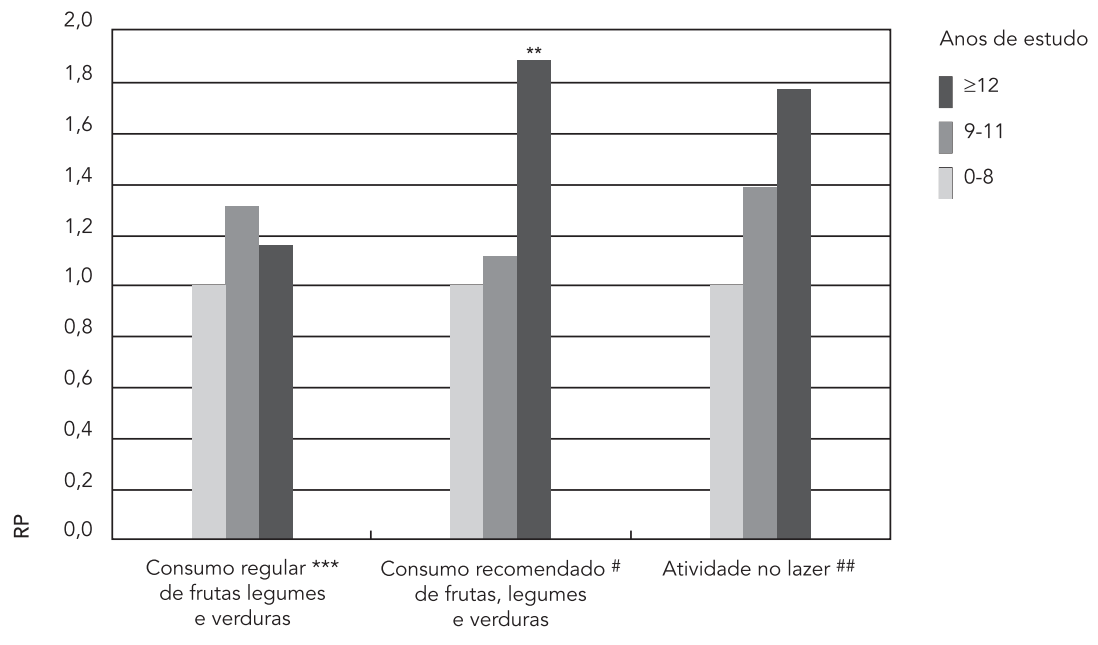

b) Mulheres

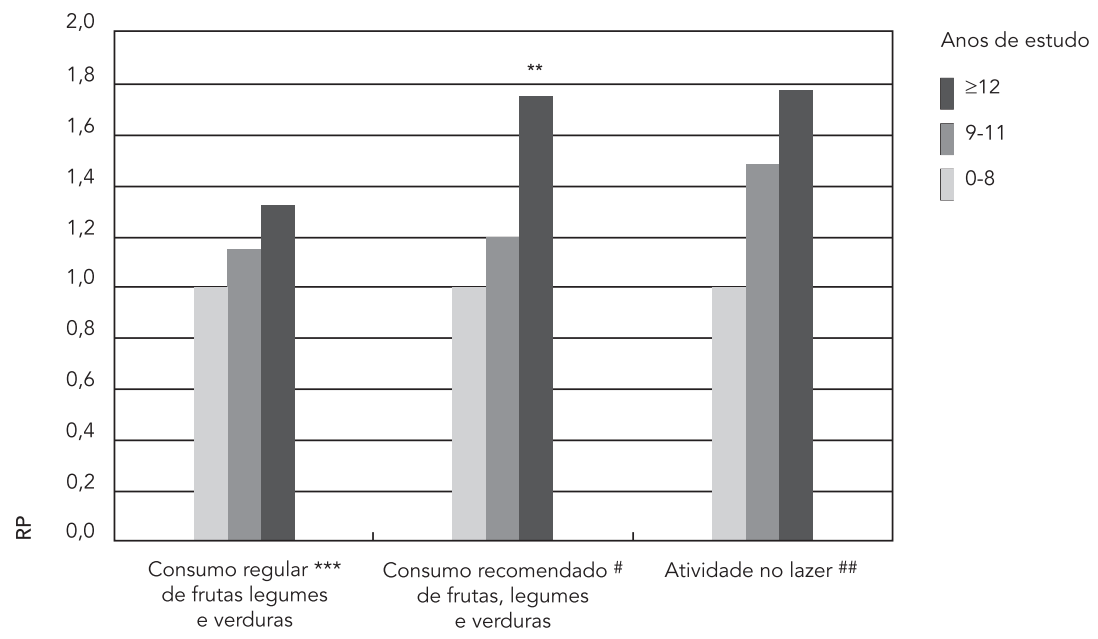

* Ponderado para ajustar a distribuição sociodemográfica da amostra VIGITEL à distribuição da população adulta de cada cidade no Censo Demográfico de 2000 (Instituto Brasileiro de Geografia e Estatística. http://www.ibge.gov.br) e para levar em conta o peso populacional de cada cidade;

** $\mathrm{p}<0,001$;

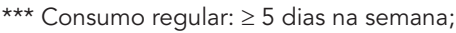

\# Consumo recomendado: $\geq 5$ por dia em $\geq 5$ dias na semana;

\#\# Atividade no lazer: atividade vigorosa (corrida, corrida em esteira, ginástica aeróbica, futebol, basquetebol ou tênis) em $\geq 20$ minutos em $\geq 3$ dias por semana ou atividade leve ou moderada em $\geq 30$ minutos em $\geq 5$ dias por semana). 
carnes na dieta da população brasileira, representando, em 2002/2003, uma participação de $13,1 \%$ na dieta 7 . Alguns estudos 13,14 têm mostrado que o aumento da escolaridade (na qualidade de proxy da renda) contribui com uma melhor dieta, não só pelo acesso à informação, mas também pelo acesso a alimentos mais saudáveis. Nossos dados mostram que a escolaridade se associou diretamente com consumo recomendado de frutas, legumes e verduras e inversamente com consumo de refrigerante com açúcar e de carnes com gordura visível em ambos os sexos.

Os achados deste estudo demonstram que os homens são mais ativos no tempo livre do que mulheres e também mais inativos no geral, o que já foi verificado em outros estudos nacionais 10,16,17. Nos Estados Unidos, também se observou tendência semelhante à encontrada neste estudo: maiores níveis de escolaridade se associam com menores níveis de atividade física no tempo livre 18,19 , possivelmente pelo maior acesso econômico aos espaços para atividade física, muitos deles privados. No Brasil, muitas mulheres ainda são responsáveis pelo trabalho doméstico, fato que pode explicar menores frequências de inatividade física em geral do que entre os homens. Este dado é corroborado com os achados da Pesquisa Nacional por Amostra de Domicílios (PNAD) de 2007 20. A frequência de inatividade física também aumenta com a escolaridade.

Os homens também apresentam mais consumo abusivo de bebidas alcoólicas, achado consistente com os de outros estudos nacionais e internacionais 10,21,22. A não associação com escolaridade pode ser reflexo da aceitação social deste hábito, sendo seu consumo estimulado em todos os níveis socioeconômicos.

No nosso estudo, as mulheres referiram com mais frequência o diagnóstico médico prévio de hipertensão arterial, o que pode ser explicado pela histórica demanda das mulheres por serviços de saúde, resultando em proporção de diagnósticos médicos maior do que de prevalência da doença em si. Achados semelhantes são relatados na Terceira Pesquisa Nacional de Saúde e Nutrição, realizada nos Estados Unidos 18. Ressalta-se que os dados aqui apresentados sobre morbidades referidas provavelmente subestimam a real prevalência de hipertensão arterial. Estudo desenvolvido em São Paulo com 2.577 indivíduos entre 30-69 anos de idade mostrou maior prevalência de diabetes em mulheres com menor escolaridade, achado que sustenta a associação encontrada no VIGITEL 2007, em que a escolaridade se associa inversamente à referência ao diagnóstico médico de diabetes, em mulheres 23 .

O VIGITEL é um estudo transversal e apresenta estimativas de fatores de risco ou proteção para doenças crônicas, caracterizando-se pela agilidade, praticidade e possibilidade de monitorar tendências na população adulta das 27 cidades brasileiras. Essas propriedades o tornam um importante instrumento de controle de fatores de risco e proteção para doenças crônicas não transmissíveis. 


\section{Resumo}

Este artigo descreve a prevalência de fatores de risco $e$ proteção para doenças crônicas não transmissíveis, a partir dos dados de 2007 do sistema de monitoramento por inquérito telefônico - o VIGITEL. Foram avaliados 54.251 adultos das capitais de estados e do Distrito Federal, Brasil. Os homens apresentaram maior frequência de hábito de fumar, de excesso de peso, de consumo de refrigerantes e de carnes com gordura, de atividade física suficiente no tempo livre, de inatividade física no geral e de consumo abusivo de bebidas alcoólicas; as mulheres, de consumo regular e recomendado de frutas, legumes e verduras. Para os homens, no nível de maior escolaridade, a probabilidade de tabagismo reduz $42 \%$, e a de consumo de carnes com gordura, 31\%; as probabilidades de excesso de peso e de inatividade física aumentam, respectivamente, $86 \%$ e $42 \%$; consumo recomendado de frutas, legumes e verduras atinge 89\%; atividade física no tempo livre aumenta para 78\%. Para as mulheres, a probabilidade de tabagismo reduz em $31 \%$, excesso de peso, $26 \%$, e consumo de carnes com gordura, 35\%; inatividade física aumenta em $76 \%$, atividade física no tempo livre, $77 \%$, consumo regular de frutas, legumes e verduras, 48\%, e recomendado, $75 \%$.

Doença Crônica; Adulto; Vigilância

\section{Referências}

1. World Health Organization. The World Health Report 2002: reducing risks, promoting healthy life. Geneva: World Health Organization; 2002.

2. World Health Organization. Preventing chronic diseases: a vital investment. Geneva: World Health Organization; 2005.

3. Malta DC, Cezário AC, Moura L, Morais Neto OL, Silva Júnior JB. A construção da vigilância e prevenção das doenças crônicas não transmissíveis no contexto do Sistema Único de Saúde. Epidemiol Serv Saúde 2006; 15:47-65.

4. Strong K, Mathers C, Leeder S, Beaglehole R. Preventing chronic diseases: how many lives can we save? Lancet 2005; 366:1578-82.

5. Secretaria de Vigilância em Saúde, Ministério da Saúde. VIGITEL 2006. Vigilância de fatores de risco e proteção para doenças crônicas por inquérito telefônico. Brasília: Ministério da Saúde; 2007.

6. Moura EC, Morais Neto OL, Malta DC, Moura L, Silva NN, Bernal R, et al. Vigilância de Fatores de Risco para Doenças Crônicas por Inquérito Telefônico nas capitais dos 26 estados brasileiros e no Distrito Federal (2006). Rev Bras Epidemiol 2008; 11 Suppl 1:20-37.

\section{Colaboradores}

E. C. Moura participou da concepção, análise e redação do artigo. S. A. Silva contribuiu na revisão bibliográfica, elaboração do manuscrito e aprovação da versão final. D. C. Malta colaborou na revisão crítica do conteúdo do artigo. O. L. Morais Neto contribuiu na aprovação final da versão final do artigo.
7. Instituto Brasileiro de Geografia e Estatística. Pesquisas de orçamentos familiares 2002-2003: análise da disponibilidade domiciliar de alimentos e do estado nutricional no Brasil. Rio de Janeiro: Instituto Brasileiro de Geografia e Estatística; 2004.

8. Secretaria de Vigilância em Saúde, Ministério da Saúde. VIGITEL 2007. Vigilância de fatores de risco e proteção para doenças crônicas por inquérito telefônico. Brasília: Ministério da Saúde; 2008.

9. Monteiro CA, Cavalcante TM, Moura EC, Claro RM, Szwarcwald CL. Population-based evidence of a strong decline in the prevalence of smokers in Brazil (1989-2003). Bull World Health Organ 2007; 85:527-34

10. Instituto Nacional de Câncer. Inquérito domiciliar sobre comportamentos de risco e morbidade referida de doenças e agravos não transmissíveis: Brasil, 15 capitais e Distrito Federal, 2002-2003. Rio de Janeiro: Instituto Nacional de Câncer; 2004.

11. Matheson FI, Moineddin R, Glazier RH. The weight of place: a multilevel analysis of gender, neighborhood material deprivation, and body mass index among Canadian adults. Soc Sci Med 2008; 66: 675-90. 
12. Monteiro CA, Conde WL, Castro IRR. A tendência cambiante da relação entre escolaridade e risco de obesidade no Brasil (1975-1997). Cad Saúde Pública 2003; 19 Suppl 1:S67-75.

13. Jaime PC, Monteiro CA. Fruit and vegetable intake by Brazilian adults, 2003. Cad Saúde Pública 2005; 21 Suppl 1:S19-24.

14. Serdula MK, Gillespie C, Kettel-Khan L, Farris R, Seymour J, Denny C. Trends in fruit and vegetable consumption among adults in the United States: behavioral risk factor surveillance system, 19942000. Am J Public Health 2004; 94:1014-8.

15. Roos G, Johansson L, Kasmel A, Klumbiené J, Prättälä R. Disparities in vegetables and fruit consumption: European cases from the north to the south. Public Health Nutr 2001; 4:35-43.

16. Monteiro CA, Conde WL, Matsudo SM, Matsudo VR, Bonsenor IM, Lotufo PA. A descriptive epidemiology of leisure-time physical activity in Brazil, 1996-1997. Rev Panam Salud Pública 2003; 14: 246-54.

17. Hallal PC, Dumith SC, Bastos JP, Reichert FF, Siqueira FV, Azevedo MR. Evolução da pesquisa epidemiológica em atividade física no Brasil: revisão sistemática. Rev Saúde Pública 2007; 41:453-60.
18. Crespo CJ, Smit E, Andersen RE, Carter-Pokras O, Ainsworth BE. Race/ethnicity, social class and their relation to physical inactivity during leisure time: results from the Third National Health and Nutrition Examination Survey, 1988-1994. Am J Prev Med 2000; 18:46-53.

19. Sternfeld B, Ainsworth BE, Quesenberry CP. Physical activity patterns in a diverse population of women. Prev Med 1999; 28:313-23.

20. Instituto Brasileiro de Geografia e Estatística. Pesquisa Nacional por Amostra de Domicílios, 2007. Rio de Janeiro: Instituto Brasileiro de Geografia e Estatística; 2007.

21. Barros MBA, Botega NJ, Dalgalarrondo P, MarínLeón L, Oliveira HB. Prevalence of alcohol abuse and associated factors in a population-based study. Rev Saúde Pública 2007; 41:502-9.

22. Centers for Disease Control and Prevention. Alcohol use among adolescents and adults: New Hampshire, 1991-2003. MMWR Morb Mortal Wkly Rep 2004; 53:174-5.

23. Goldenberg P, Sckenkman S, Franco LJ. Prevalência de diabetes mellitus: diferenças de gênero e igualdade entre os sexos. Rev Bras Epidemiol 2003; 6:18-28.

Recebido em 28/Jul/2009

Versão final reapresentada em 12/Ago/2010

Aprovado em 15/Out/2010 